\title{
El uso de los medios desde los modelos del currículum
}

\author{
Antonio Bautista
}
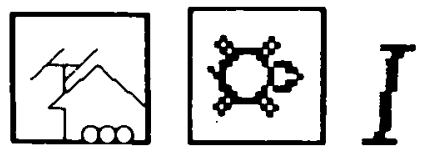

El uso de los medios de comunicación y las tecnologías de la información en la escuela está experimentando una auténtica convulsión desde la incorporación del ordenador al escenario escolar. La anterior reforma educativa emprendida en nuestro país se alumbró bajo los prometedores auspicios de los medios audiovisuales y ya entonces se levantaron voces, bien catastrofistas, bien entusiastas vaticinando en ambos casos consecuencias que revolucionarian los modos de enseñar en la escuela. El paso de los años ba demostrado que ambas premoniciones fueron inexactas y que la escuela $\mathrm{ba}$ permanecido relativamente impermeable a la revolución tecnológica del mundo exterior. La aparición del ordenador ba vuelto a suscitar una polémica de parecidas características, afortunadamente superada, aunque es cierto que el entorno informatizado -o aún más el entorno bipermedia que se avecina - al que inevitablemente se aboca la sociedad de boy, supone tales cambios cualitativos en el modo de organizar el conocimiento y por tanto la impartición del conocimiento, que la escuela no podrá por menos que adoptar una actitud emprendedora respecto a las nuevas tecnologías como parte integrante del curriculum.

Ante las tres teorías del currículum que se distinguen en estos momentos (Carr y Kemmis, 1988; Kemmis, 1988) contemplamos tres tipos de usos de los medios:

a) Usos transmisores/reproductores

La teoría del currículum que orienta este tipo de uso es la técnica o tecnológica. Sus características básicas son:

- La centralización de la selección y organización de contenidos,

- La linealidad de su diseño (objetivos $\rightarrow$ contenidos $\rightarrow$...),

- Existe una separación entre teoría/planificación y la práctica/ejecución, donde el profesor es un reproductor y ejecutor de las orientaciones que le llegan diseñadas desde la administración central de educación de su país. 
- La preocupación por lo observable y medible y por la búsqueda de eficientismo social y laboral.

En este contexto, el uso que se hace de los medios es para presentar informaciones, temas, mensajes, etc. Soportados en programas, películas... según llegan al aula vía administrativa, vía editorial, etc. A su vez, estas materias y temas implícitos en los materiales difundirán una cultura determinada y darán una perspectiva del hombre y de la sociedad que estará de acuerdo con quienes seleccionan y organizan esos contenidos.

El papel de profesores y alumnos es el de ejecutar las demandas de esos materiales de paso. Torres Santomé $(1987-88,1989)$ analizó ambos roles en el uso que normalmente se hace de los libros de texto. Así, cuando se presenta a los alumnos unos materiales que no favorecen la interacción y la toma de decisiones, cuando no se fomenta la consulta de otras fuentes de información, cuando esos medios y materiales son necesarios y suficientes para aprobar los exámenes (pues contienen las preguntas y las respuestas), entonces se anula el sentido crítico y la reflexión que son necesarios para un desarrollo personal, social y cultural de tales alumnos.

Las tareas diseñadas, incluidas en películas, software educativo... y que se programan con antelación, normalmente son difíciles de modificar cuando surge alguna incidencia en los procesos de enseñanza y de aprendizaje (dificultades de comprensión, detener el programa o película en un momento determinado para codificar semánticamente una información o mensaje, etc.) y por lo tanto, son consideradas cerradas e inflexibles.

Este uso está justificado para desarrollar tareas que lleven a un aprendizaje memorístico o a la adquisición de rutinas cognitivas (realización de ejercicios, aplicación de fórmulas...). Es decir, tareas que tengan un bajo nivel de ambigüedad (que comuniquen a los alumnos cuál ha de ser la actuación exacta y la forma de realizarla) y poco riesgo (que los alumnos sean capaces de hacer frente a esa tarea presentada).

Como los profesores y alumnos no reflexionan ni participan en la selección de contenidos, ni en su organización, ni en las estrategias utilizadas para su presentación, existe la posibilidad de que asuman y reproduzcan valores, intereses, ideologías, etc., que están ocultos, por trabajar unos temas y no otros, por presentarlos de una forma y no de otra, etc.

\section{b) Usos prácticos/situacionales}

Los aspectos básicos de la teoría del currículum práctica o interpretativa que orienta este tipo de uso, son los siguientes:

- Se parte de un análisis e interpretación de situaciones concretas de enseñanza y de una comprensión de los significados generados por profesores y alumnos en las aulas.

- Los procesos de enseñanza se consideran únicos, multidimensionales e irrepetibles, $y$, consecuentemente, difíciles de predecir con antelación, porque a los planteamientos y diseños que hace el profesor antes de la clase, hay que añadir todos los elementos y circunstancias no previstas que se generan en los procesos de interacción que tienen lugar en las aulas.

- Los equipos de profesores organizan contenidos y diseñan tareas que llevan a un trabajo coherente y lleno de significado para los alumnos.

- Son, pues, diseños abiertos que tienen presente las dimensiones contextuales de la enseñanza.

Estos aspectos básicos implican el uso de los medios que vaya precedido por 
el análisis y la comprensión de los significados, construidos éstos por grupos concretos de profesores y alumnos.

Partiendo del conocimiento de esa realidad el profesor o grupo de profesores, tendrá más posibilidades para diseñar tareas comprensivas y llenas de sig. nificado para los alumnos. Tareas coherentes que pueden estar inscritas dentro de situaciones que se viven como problemáticas o en los proyectos de trabajo.

En esta forma de uso, los equipos de vídeo, ordenador, ..., además de ser herramientas que utilizan los alumnos para comprobar hipótesis, simular procesos, ejecutar planes, etc., con el fin de resolver problemas o desarrollar proyectos, proporcionan y hacen posible el empleo de diversos sistemas de representación.

Entendemos por sistemas de representación las formas o recursos que posee y utiliza un sujeto para expresar/comunicar ideas, experiencias, hechos, etc., de la vida. Como todo sistema, contienen una serie de elementos que mantienen determinadas relaciones entre sí (lenguajes de programación, códigos icónicos, estructura narrativa de la imagen dinámica...)

Consideramos que un sujeto comprende unos hechos, mensajes... cuando les da significado, y esto sucede cuando los representa en los diferentes sistemas que posee. Cuantos más sistemas de representación posea más significado les dará (más puntos de vista tendrán de lo observado), pues el pasar un mensaje, concepto, objeto... a otro sistema requiere:

- hacer un análisis de los aspectos relevantes que constituyen ese objeto, hecho... así como sus relaciones. De esta forma puede:

- establecer la correspondencia entre cada uno de los elementos constitutivos del objeto o hecho a representar (y sus relaciones) con los elementos (y sus relaciones) que constituyen el sistema donde se va a representar.

Este análisis es más profundo cuando el sistema de representación elegido contiene unos elementos más alejados de la realidad o grado cero de representación, como ocurre con los lenguajes de ordenador, notas musicales... Estos son los sistemas de representación proposicionales, y a los procesos cognitivos que llevan a dar más significativamente a unos objetos o hechos del contexto mediante el paso de un sistema de representación analógico a otro proposicional los consideramos como razonamientos en profundidad.

(Ver esquema adjunto sobre sistemas de representación).

A su vez, cuando un sujeto ha representado un hecho, objeto, estado, interno, en dos o más sistemas, entendemos que puede pensar en ese hecho, objeto... de forma dialógica, estableciendo una "conversación interna» entre las diferentes representaciones de la misma cosa. Esto supone unos beneficios para -el sujeto, pues va generando un significado progresivo en los ciclos de reflexión y deliberación entre las diferentes representaciones. Así, un objeto o concepto puede tener un nivel $\mathrm{A}$ de significado en los diferentes sistemas, pero la reflexión, comparación, análisis... efectuadas en uno de ellos, puede llevar a generar un significado A1, superior a A, en otro sistema. Este nuevo nivel de significado A1, mediante la reflexión, deliberación, interna... puede llevar a otro significado A2, superior a $A 1$... Es decir, la posibilidad de estos razonamientos entre representaciones en paralelo de una cosa, es análoga a la probabilidad de realizar razonamientos entre varios sujetos cuando están relacionados por unos procesos de diálogo.

Estos argumentos justifican que, al usar los medios de forma comprensiva/situacional, los consideremos como recursos:

- que permiten realizar representaciones, 
- que obligan y ayudan a representar problemas y encontrar más fácilmen. te sus soluciones,

- beneficiosos, pues tales representaciones constituyen estructuras cognitivas que posibilitan enfrentarse eficientemente a fuentes de información ambiguas,

- ventajosos, al permitir aprender y utilizar sistemas de representación que son básicos para desarrollar el pensamiento y para interpretar, entender y relacionarse con el contexto social, físico y cultural.

Las tareas escolares efectuadas con este tipo de medios son abiertas, tienen en cuenta las dimensiones contextuales y procesuales de la enseñanza, y consecuentemente, pueden ser modificadas según surjan elementos no previstos generados en el proceso y dinámica del grupo.

El uso de ordenador, vídeo, etc., permite realizar tareas comprensivas y expresivas o aquéllas que permiten desarrollar problemas/proyectos que no tienen una única solución ni una única forma de resolverlos. Un ejemplo de estas tareas que implica el uso del equipo de vídeo y del lenguaje cinematográfico o de la imagen móvil es «que los alumnos, por grupos, editen un vídeo de unos quince minutos donde se expresen las características y condicionantes geográficos, políticos, culturales... de un movimiento literario, evento histórico, etc.»

Evidentemente, son tareas con un alto nivel de ambigüedad y de riesgo, pero que ayudan a los alumnos a construir significados sobre los contenidos, mensajes o informaciones que se les hayan presentado en el enunciado de tales proyectos, en los debates para desarrollarlos...

L.os grupos de profesores diseñan estas tareas partiendo de la situación de sus grupos de alumnos, de los significados generados en sus aulas y de la reflexión y problematización de los contenidos que se van a trabajar. Esto significa que tendrán la oportunidad de identificar y ser conscientes de los valores que se están reproduciendo y de los intereses que se están desarrollando.

\section{c) Usos críticos/transformadores}

Algunos aspectos básicos de la teoría crítica del currículum que orienta este tipo de usos son:

- Entender que el currículum está cultural, social, económica, políticamente, etc., determinado (Apple, 1986; Popkewitz, 1987; Kemmis, 1988;...).

- Defensa de la reflexión y deliberación crítica sobre la práctica para mejorarla, transformando a su vez los códigos y principios que orientan la selección de contenidos, su organización... y que abocan a generar desigualdades sociales, dificultades en la enseñanza... (Carr y Kemmis, 1988).

- Preocupación por el desarrollo profesional del profesor que es considerado como un orientador, crítico y transformador (Elliot, 1986).

- Relación estrecha y simultánea entre teoría y práctica (Carr y Kemmis, 1988).

Estas características justifican que, en este tipo de uso, los medios se utilicen como elementos de análisis, reflexión, crítica y transformación de prácticas de enseñanza y de mensajes e informaciones que son portadoras de valores no deseados o presentaciones de posturas del saber/vida que no responden a la ver$\mathrm{dad} / \mathrm{realidad}$.

Este es el uso que se hace del sistema vídeo cuando se utiliza como tercer punto de conocimiento (Hull, 1986) para aumentar la comprensión de lo que sucede en espacios educativos y para controlar la subjetividad de las interpretaciones que hacemos de procesos (observables y no observables) del aula. La ob- 
servación, reflexión, discusión, etc., sobre estas grabaciones permitirá identificar $y$ entender dificultades $y$, posteriormente, resolverlas mediante transformaciones de la práctica.

Este es también el uso que se hace del ordenador cuando un profesor utiliza ficheros «eco" para registrar todos los procesos y operaciones que han seguido los alumnos para resolver un problema o desarrollar un proyecto de trabajo utilizando un lenguaje de programación de ordenadores. (Los ficheros "eco" son aquellos grupos de instrucciones que aparecen registrados en la impresora o se graban magnéticamente en un disco flexible o duro, y que se generan mediante una copia simultánea de todas las órdenes, informaciones o datos que un usua. rio introduce en el ordenador durante un tiempo de trabajo).

Tales ficheros serán elementos de análisis y reflexión de profesores y alumnos para entender las estrategias de resolución seguidas, interrogarse por la frecuencia de utilización de unas y por la ausencia de otras, cuestionar los significados que han dado a unas informaciones o materias objeto de aprendizaje que estaban incluidas en el enunciado de esos proyectos, etc...

Asímismo dentro de este uso, alumnos y profesores son considerados como seres analíticos, reflexivos, constructores y transformadores de prácticas e informaciones que lleguen al aula y que no respondan a unos valores deseados y a unos intereses legitimados.

Es un uso de los medios pensado y utilizado en ámbitos de formación del profesorado donde la enseñanza es considerada como algo que tiene lugar en espacios únicos, irrepetibles y difíciles de prever con antelación. Como consecuencia, el profesor, más que un.simple dominador de competencias, debe saber además cómo utilizarlas, es decir, poseer metacompetencias que le permitan, además de ser críticos, diagnosticar situaciones conflictivas, procesar informaciones, dar respuestas a esas dificultades... (Gimeno, 1983; Peters, 1987; Contreras, 1987).

Este uso de los medios en la formación del profesorado:

- Proporciona elementos de análisis y deliberación sobre la práctica desarrollada por el profesor con el fin de entenderla y mejorarla,

- Presenta entornos flexibles e interactivos con capacidad de simular aspectos de la realidad educativa, que ayuden al profesor/alumnos a reflexionar, tomar decisiones, etc.

- Permite conocer mejor el funcionamiento y la construcción del conocimiento práctico del profesor según lo definen Pérez Gómez, (1988), Pérez y Gimeno (1988), y Shulman (1987) entre otros.

Es, pues, un uso de los medios preocupado por mejorar y transformar la práctica del aula y por desarrollar profesionalmente a los profesores.

\section{$¿ Q U E$ USOS SE CONTEMPLAN EN LAS ORIENTACIONES PROPORCIONADAS POR DIFERENTES ESTAMENTOS Y EN LOS DIFERENTES AMBITOS EDUCATIVOS DE NUESTRO PAIS?}

Haremos mención a los estamentos y ámbitos que figuran en los siguientes interrogantes:

a) De los tres usos señalados, ¿cuál es el que predomina en las recomendaciones y materiales desarrollados por las editoriales y casas comerciales?

b) ¿Qué filosofías sobre usos de los medios se defienden en el Programa de Nuevas Tecnologías del MEC? 

las aulas?

c) ¿Cómo se usan los medios en nuestro contexto educativo más próximo:

d) Relacionado con el punto b), ¿Qué uso se preconiza en los proyectos destinados a integrar las Nuevas Tecnologías en las diferentes Comunidades Autónomas? (Proyectos Telegal, Vasco, Catalán, Alhambra...)

e) ¿Cómo se utilizan estos medios en las Escuelas universitarias de Formación del Profesorado de EGB y en los Cursos de Aptitud Pedagógica (CAP) organizados por los Institutos de Ciencias de la Educación para formar a los futuros profesores de Bachillerato?

f) ¿Qué usos defienden y proponen los grupos de Renovación Pedagógica sobre Nuevas Tecnologías?

g) Finalmente, ¿qué usos de los medios se recomiendan en el Libro Blanco y en los Diseños Curriculares Base que recientemente ha publicado el MEC para su discusión?

Aunque no hay datos exhaustivos respecto al uso de los medios en todos los ámbitos educativos señalados, daremos respuesta a los anteriores interrogantes con las informaciones que iremos mencionando oportunamente.

a) Respecto al primer interrogante, observamos que el uso del vídeo que recomiendan diferentes casas comerciales es el transmisor-reproductor, pues uno de sus propósitos es vender los «videocasettes» que han elaborado sobre diferentes temas con el fin de que los profesores lo utilicen para comunicar a sus alumnos los contenidos implícitos en tales películas (sobre «energía», sobre «nubes y precipitaciones», sobre «expresión escrita»...)

Algunas de estas casas comerciales adjuntan al «videocasette» una guía o vídeo-guía donde se describen los objetivos, los contenidos y unas sugerencias sobre actividades para después de la proyección.

Evidentemente, un buen número de profesores utilizan estos vídeos como sustitutos de su comunicación o explicación oral, y después de «ponerles la película a los alumnos», recurren a las actividades contempladas en el vídeo-guía.

En este uso transmisor-reproductor, queda implícito con claridad el tipo de control que se establece sobre el profesor y sobre los alumnos. Según Apple (1986), hay que distinguir entre el control simple (decir a alguien lo que tiene que hacer), el control técnico (incrustado en la estructura física del trabajo, por ejemplo, cuando un trabajador inserta un programa en una máquina que dirige el trabajo y relega la función de los sujetos a ser meros acompañantes del proceso), y el control burocrático (que es menos visible, pues está incorporado en las relaciones sociales jerarquizadas de los centros de trabajo).

Podemos establecer cierto paralelismo entre el tipo de control técnico expuesto por Apple y los tipos de materiales y usos de medios en el aula que preconizan algunas casas comerciales. Esta analogía se da cuando para un área escolar se adquiere un conjunto de materiales, que incluyen una relación de objetivos y actividades para profesores y alumnos, que les evitan aprender y utilizar unos sistemas de representación, asumir otros puntos de vista sobre esos contenidos...

Esto significa que, si los usos están determinados/condicionados por el control técnico ejercido por los materiales, entonces los beneficios culturales y cognitivos de los medios y, consecuentemente, las diferencias sociales, están condicionados entre otros aspectos, por el control técnico, o códigos del currículum, que se hace desde unos tipos de usos que, a su vez, están determinados por la adquisición de un tipo de material. 
Algo parecido sucede con los usos propuestos por algunas editoriales de software educativo (programas de ordenador dirigidos a la enseñanza). Normalmente estas editoriales. venden un diskette, junto a un manual, con el propósito de que los alumnos aprendan unos contenidos del currículum (los gases, circuitos de corriente continua...). También de forma análoga a los materiales de vídeo, el libro proporciona informaciones sobre el tema y facilita una relación de actividades (en muchas ocasiones cerradas o lo que es lo mismo con una única respuesta válida).

A su vez, los diskettes contienen una serie de programas que cargados y ejecutados en el ordenador, convierten a este equipo en un «profesor» que dirige los procesos de aprendizaje de los alumnos, proporcionando unas informaciones y formulando unas preguntas que deben ser respondidas a través del teclado del ordenador.

El alumno difícilmente puede alejar sus procesos de pensamiento de este con. texto informático concreto, generado por un uso transmisor del ordenador, pues está condicionado por los datos, elementos e interrogantes que proporciona este equipo electrónico, normalmente, a través de la pantalla.

b) Los proyectos Atenea y Mercurio, que iniciaron su camino de forma separada en 1985, se integraron en el Programa de Nuevas Tecnologías de la Información y de la Comunicación (PNTIC) en 1987. Su ámbito de aplicación es el territorio MEC.

En la versión resumida del proyecto Atenea presentada en 1985, observamos la siguiente distinción de usos del ordenador:

El ordenador como berramienta para el alumno (pág. 19 y ss.) donde se utiliza con la intención de desarrollar su capacidad creativa y el aprendizaje por descubrimiento. Para ello, se propone la elaboración de programas educativos y juegos, y el aprendizaje de un lenguaje de programación, principalmente Logo. Respecto a la línea de trabajo a seguir, se sugiere un cambio en el uso convencional del ordenador en la enseñanza.

El ordenador como herramienta para el profesor (pág. 24 y ss.), utilizado con dos propósitos: para alfabetizarse informáticamente y para mejorar su metodología. Las orientaciones que se dan sobre cómo usar este equipo electrónico, son múltiples, pues van desde utilizar programas instructivos elaborados por casas comerciales y grupos de trabajo hasta el uso de lenguajes informáticos y programas de aplicación (procesadores de texto, bases de datos...)

Desde nuestro punto de vista, consideramos que el proyecto Atenea materializa una propuesta abierta que contiene orientaciones sobre dos tipos de usos de los medios: transmisores/reproductores y situacionales/comprensivos.

En el documento presentado por el Programa de Nuevas Tecnologías en 1988 , con el fin de dar a conocer las realizaciones y actuaciones previstas en los proyectos Atenea y Mercurio a la comunidad educativa, observamos que no hay unas orientaciones explícitas sobre el uso de estos medios aunque sí subyacen los dos tipos de usos recogidos en el documento que hemos comentado anteriormente. Esta afirmación la basamos en estos tres grupss de informaciones:

1. Se dota a los Centros de Profesores de un amplio número de películas de vídeo y programas educativos de ordenador sobre temas de todas las áreas curriculares: Ciencias Naturales... Matemáticas...

- La propuesta de producción de vídeos educativos, bien en la unidad cen- 
tral del programa de NTIC, bien a través de otras instituciones (Facultad de Ciencias de la Información, Facultad de Filosofía y Ciencias de la Educación...) También se manifiesta que

«se impulsará a empresas privadas para la producción de vídeos didácticos mediante ayudas, subvenciones y acuerdos de coproducción...». (Programa NTIC, 1988, p. 32).

- Idem en la propuesta de producción de programas educativos de ordenador, bien por el propio MEC, bien

«incentivando a empresas editoriales y de software para la producción de programas educativos mediante subvenciones y créditos privilegiados...». (Programa NTIC, 1988, p. 20).

2. Dotando a los centros experimentales con equipos que pueden llevar a usar los medios de forma comprensiva:

- En el caso del proyecto Mercurio, se proporcionan equipos de grabación (cámaras, magnetoscopios portátiles, trípodes...) equipos de mezcla de sonido, equipos de iluminación...

- En el caso del proyecto Atenea, dotando a los centros de intérpretes y compiladores de lenguajes (Logo, Pascal...), programas de aplicaciones.

3. Formando a los profesores desde dos estrategias educativas:

- Usando programas instructivos de vídeo y ordenador,

- Entrenando a los profesores en análisis de documentos, análisis de la imagen, definición de posibilidades de los sistemas de vídeo y ordenador en metodologías activas...

Consideramos que en esta doble orientación sobre el uso de los equipos tecnológicos, reproductor y comprensivo, faltan consideraciones sobre la utilización del vídeo y del ordenador en el aula y en la formación del profesorado que permitan:

- Comprender mejor el tipo de significados y negociaciones que se realizan en esos lugares de enseñanza y de aprendizaje,

- Observar las situaciones definidas como «normales» en nuestras aulas, y sobre todo,

- Reflexionar y deliberar sobre los problemas y dificultades que se generan en la interacción que tiene lugar en los espacios de enseñanza, para entenderlas y transformar las prácticas y actividades incoherentes que lleven a los alumnos al aburrimiento y al hastío.

Pero, ¿cómo llegan a los centros escolares estos planteamientos y orientaciones?, ¿cómo se interpretan y desarrollan en las aulas?

c) Vamos a intentar dar respuesta a estos interrogantes utilizando, principalmente, el «Informe de progreso» sobre el proyecto Atenea que ha publicado recientemente el Programa de Nuevas Tecnologías de la Información y Comunicación (PNTIC). En el tercer apartado de dicho informe, titulado «El proyecto Atenea en las aulas», recoge las respuestas dadas por los profesores cuando se les preguntó mediante un cuestionario «qué funciones atribuían al ordenador».

De los 1.210 profesores no coordinadores implicados en el proyecto Atenea que fueron invitados a ordenar nueve categorías o funciones posibles que tiene el ordenador en el aprendizaje (categorías que iban desde usar este equipo electrónico para motivar al alumno hasta utilizarlo para realizar actividades propuestas, resolver problemas, evaluar el aprendizaje...), aproximadamente un $50 \%$ no señalaron todas las categorías en la ordenación que efectuaron. 
La categoría más veces elegida en primer lugar $(40,83 \%$ de las respuestas) fue la atribución y uso del ordenador "como elemento motivador». La segunda categoría con el 25,38\% veces elegida en primer lugar fue el uso del ordenador «para desarrollar actividades prácticas». Los usos de este recurso informático menos valorados fueron «para hacer actividades recreativas» $(1,73 \%)$ «para evaluar los aprendizajes» $(1,82 \%)$ y «para resolver problemas en actividades prácticas» $(4,88 \%)$.

De estos datos, (¿cuáles habrían sido si no se hubiesen proporcionado las nueve categorías previas y las tuviesen que haber definido y explicado los profesores?) interpretamos que de todas las orientaciones proporcionadas desde el PNTIC sobre los usos del ordeandor, llegan con más claridad, o se entienden mejor, los que hemos denominado transmisores/reproductores, pues un porcentaje alto de los profesores implicados en el proyecto Atenea, utilizan este medio para desarrollar tareas sencillas, con bajo nivel de dificultad y sin tener presente el contexto, como sucede, bien cuando utilizan el ordenador para motivar, bien cuando lo usan para desarrollar actividades prácticas que no sean resolver problemas...

También llegamos a esta interpretación sobre el uso transmisor que se hace del ordenador en las aulas al responder a este interrogante, ¿qué significa utilizar el ordenador como elemento motivador? Mediante una entrevista realizada a seis profesores implicados en el proyecto Atenea, llegamos a entender que este uso motivador estaba originado por la predisposición favorable y positiva que tienen los alumnos a realizar unas actividades con ordenador que, posteriormente, les llevarán a asumir unos contenidos de la cultura. Sobre esta función del ordenador surgen dos nuevas dudas:

- ¿Este uso como elemento motivador, se debe a una característica intrínseca o esencial del ordenador o es algo peculiar de todos los medios cuando se emplean para realizar actividades en el aula?

Desde nuestro punto de vista, entendemos que esta dimensión motivadora es algo propio de todos los medios y que más que una cualidad intrínseca, el entusiasmo que suscita se debe al efecto «novedad» del uso de un elemento nuevo en las prácticas cotidianas, pues los profesores entrevistados manifiestan que «según pasaba el tiempo parecía como si los chicos se cansaran de trabajar con este recurson.

- ¿Qué tareas se realizan cuando se usa el ordenador como elemento motivador? A esta cuestión cinco de los seis profesores entrevistados respondieron que eran actividades orientadas por un programa educativo de ordenador. El sexto profesor que la función motivadora carecía de sentido y que prefería hablar del uso del ordenador como medio para resolver problemas, para ello planteaba tareas y los alumnos utilizando un lenguaje, que previamente habían aprendido, se dirigían a este equipo electrónico para ensayar un plan, comprobar una solución... e intentar resolverlo.

$\mathrm{Al}$ margen de nuestras dudas sobre la validez de estas informaciones, pues son el resultado de la interpretación que cada profesor hace de su práctica cuando responde a las preguntas del cuestionario (hubiese sido mejor obtener esas informaciones mediante observación directa de las aulas), entendemos que el uso del ordenador que predomina es el transmisor/reproductor.

d) No tenemos información sobre las orientaciones respecto al uso de los medios que se proporcionan en todos los proyectos diseñados por las diferentes Comunidades Autónomas con competencias en educación y que tienen como 
propósito integrar las nuevas tecnologías en los currículums. Nos referiremos pues a los proyectos de los que poseemos información.

Así, respecto al Plan Vasco de Informática Educativa; se pueden aplicar los comentarios que hemos realizado sobre el proyecto Atenea, pues recoge la filosofía de éste respecto a las orientaciones sobre las funciones y usos del ordenador: «utilización del ordenador como herramienta», «utilización del ordenador como soporte para ejecutar programas educativos (E. A. O.), «utilización del ordenador como auxiliar burocrático»... Respecto al Plan Alhambra, según el diseño del plan de actuación para la introducción de la informática en los niveles de enseñanza no universitaria que ha realizado la Consejería de Educación y Ciencia de la Junta de Andalucía, observamos una ausencia de orientaciones sobre los posibles usos que se puede hacer del ordenador. Sin embargo, percibimos un excesivo énfasis en la preparación técnica del profesorado y una falta de sugerencias sobre las posibilidades y estrategias de integración de estas tecnologías de la información en las diferentes áreas de contenidos.

Estos aspectos del Plan Alhambra junto a los objetivos generales que proponen para la EGB y Enseñanzas Medias, nos llevan a considerar que el propósito de este proyecto es introducir una asignatura de Informática en los niveles de Enseñanza Básica y Secundaria. Sobre el tipo de uso que se hará del ordenador, se manifiesta que se potenciará la creación de material didáctico adecuado. Esperamos que la estructura de este material y la utilización que se haga de él no esté orientada por una filosofía exclusivamente reproductora/transmisora.

e) Sobre el uso que se hace actualmente de los medios en las Escuelas Universitarias de Formación del Profesorado y en los ICES, no tenemos información.

En las publicaciones sobre el uso del vídeo en la formación del profesorado durante las décadas de los 60 y 70, predomina el uso del Circuito Cerrado de Televisión (CCTV) en ámbitos de microenseñanza. El modelo de formación del profesorado que orienta este uso del vídeo es el basado en el desarrollo de competencias o acciones complejas. Este modelo considera que el aprendizaje de estas competencias se realiza mejor cuando se dividen en conductas simples o destrezas (cada una queda definida por un conjunto de conductas observables), aprendiéndose y perfeccionándose cada una de ellas por separado a través de la técnica de la microenseñanza.

El papel del vídeo dentro de estas técnicas es presentar «modelos filmados» para que sirvan como ejemplos o patrones a imitar por futuros profesores en el proceso de moldeamiento y adquisición de destrezas específicas. Este uso del vídeo dentro del CCTV surgió a partir de los estudios de Bandura, Ross y Ross (1963) cuando concluyeron que los modelos filmados son tan eficaces como los de la vida real en los procesos de moldeado de destrezas.

Sobre el uso de los medios en este modélo de formación del profesorado, queremos indicar que:

1. Es un uso transmisor/reproductor, pues tiene como fin reproducir rasgos relevantes de comportamiento de profesores «modelos» que se consideran necesarios para desarrollar una enseñanza de calidad.

2. Se olvida que en la enseñanza intervienen multitud de variables que hacen que se produzcan situaciones no previstas que es preciso abordar en el proceso. Esto significa que hay que usar los medios para desarrollar metacompetencias (Gimeno, 1983) y no destrezas en los profesores.

3. Es necesario identificar las dificultades que se producen en nuestras au- 
las, analizarlas críticamente, transformarlas proporcionando alternativas de mejora, llevarlas a la práctica, etc. Los medios usados de forma comprensiva y crítica tienen un buen papel en estos planteamientos sobre la formación del profesorado.

f) Los grupos de renovación pedagógica preocupados por la incorporación de las Nuevas Tecnologías de la Información y de la Comunicación (NTIC) en el mundo de la educación, se preocupan principalmente por desarrollar materiales y estrategias de enseñanza que ayuden a un usuario a conocer y a utilizar elementos básicos relacionados con esos medios (lenguajes de programación, lenguaje cinematográfico, procesadores de texto, bases de datos, edición de vídeos educativos...)

Normalmente tampoco estos grupos han realizado planteamientos serios o iniciado líneas de investigación que permitan conocer los procesos que se generan en las aulas cuando unos alumnos trabajan de una forma determinada con esos medios e intentar entender cómo asumen los contenidos implícitos en esas actividades, qué ventajas supone a los profesores y a los jóvenes esa manera de utilizar los recursos tecnológicos...

Respecto al tipo de uso que se hace de los medios, predomina el uso transmisor-reproductor que definimos líneas atrás. Esto se puede comprobar analizando las experiencias que aparecen publicadas en las revistas editadas por algunos de estos grupos. Buen número de estos trabajos giran en torno al diseño de unos materiales por parte del profesor o de alguna casa editorial (vídeos y software educativo...) para que, posteriormente, los alumnos los ejecuten y aprendan los conceptos implícitos en tales materiales.

Normalmente, en las experiencias publicadas no se expresan cuáles son las preocupaciones o problemas que han llevado a grupos de profesores y de alumnos a realizar ese estudio, ni el contexto físico, social y cultural donde se realiza, ni el punto de vista de los participantes en esa investigación.

Estas ausencias en las experiencias realizadas y publicadas por algunos «grupos de renovación visual e informática en educación» significan:

- que no se ha hecho un uso comprensivo y situacional de los medios, y

- que consideran que se pueden generalizar esas experiencias a otros contextos diferentes.

Entendemos pues, que en estos trabajos se hace un uso transmisor de los medios, entre otras razones, porque olvidan la perspectiva ecológica (que dice que el comportamiento humano está influido por el medio físico, social y cultural donde se realiza), y fenomenológica (para entender el comportamiento humano hay que tener en cuenta el marco de referencia que cada sujeto usa para interpretar y dar sentido... a todo lo que le rodea), que es preciso tener presentes en toda investigación educativa.

g) No queremos terminar estas reflexiones, sin hacer referencia a los usos de los medios que se recomiendan en los documentos sobre la reforma de la enseñanza publicados recientemente por el MEC para su discusión.

Analizando estos documentos, observamos que en el Libro Blanco de la reforma no se explicita ni el papel de los medios en la enseñanza ni se proporcionan orientaciones sobre los usos que se puede hacer de ellos.

Donde sí encontramos sugerencias para utilizar los medios es en los Diseños Curriculares Base (DCB). En los DCB de Educación Primaria, las recomendaciones son variadas. Van desde considerarlos como fuentes de información para trabajar contenidos, por ejemplo en el área «Conocimiento del Medio», 
«Deben aprovecharse diferentes fuentes de información... tanto procedentes del entorno... como vinculados a las diversas tecnologías de la información, vídeo, pren. sa, magnetófono, cine, ordenador.» (DCB Educación Primaria, 1989, p. 133).

hasta usarlos como soportes para ejecutar materiales audiovisuales y programas de ordenador con fines educativos. Un ejemplo de estas orientaciones las encontramos en el área de Matemáticas

«los materiales audiovisuales... pueden consistir en presentaciones históricas e ilustraciones que sirvan de apoyo o de punto de partida de actividades matemáticas... Asimismo, el ordenador puede facilitar la adquisición y consolidación de conceptos y destrezas matemáticas. Son aconsejables los programas que se adaptan al ritmo de aprendizaje del alumnado que interactúa con ellos, proponiendo distintos tipos de ejercicios en relación con los errores que se comentan» (DCB Educación Primaria, 1989, pp. 412-413).

Sobre estas recomendaciones de uso de programas de ordenador queremos manifestar que aunque se exprese que han de adecuarse al ritmo de los alumnos (todos lo hacen cuando formulan preguntas y esperan impasiblemente las respuestas de los usuarios...) y a pesar de ser capaces de presentar actividades que ayuden a superar los errores cometidos, desde nuestro punto de vista, estos planteamientos de uso se encuadran dentro del tipo transmisor/reproductor.

Algo análogo sucede en los DCB de Educación Secundaria Obligatoria. Así en las orientaciones generales sobre actividades a realizar en el área de Ciencias Naturales, se recomienda el uso de diapositivas, vídeos... y sobre la utilización del ordenador se manifiesta que se están

«diseñando materiales didácticos de interés cuya eficacia en las aulas merecería ser contrastada.» (DCB Enseñanza Secundaria Obligatoria I, 1989, p. 161).

Asímismo, en el área de «Expresión Visual y Plástica» de esta etapa de enseñanza obligatoria, echamos de menos una propuesta de objetivos y un uso de los medios orientados a hacer un análisis crítico de informaciones y publicidad estática y dinámica soportada en el lenguaje usado por estos medios.

Finalmente queremos indicar que aunque son frecuentes este tipo de orientaciones transmisoras/reproductoras sobre el uso de los medios, también en los DCB de Educación Secundaria Obligatoria se contemplan recomendaciones sobre usos comprensivos/situacionales, por ejemplo en el área de «Geografía, Historia y Ciencias Sociales», se presenta al ordenador como un recurso didáctico que permite:

«consultar con rapidez muchos datos, procesarlos con agilidad, realizar cálculos estadístico-matemáticos más o menos complejos, elaborar gráficos, mapas e imágenes... Por otra parte, y en un grado más elevado de dificultad, el ordenador ofrece la posibilidad de comprobar de manera inmediata la validez de las hipótesis que los alumnos pueden plantear ante determinados problemas, controlando sucesivamente diferentes variables.» (DCB Educación Secundaria Obligatoria, I, 1989, p. 339).

Así pues, aunque desde nuestro punto de vista es insuficiente el énfasis que se pone en este uso comprensivo de los medios, concluimos este análisis indicando que en las orientaciones sobre el uso de los medios propuestas en los Diseños Curriculares Base, predominan las sugerencias hacia los usos reproductores/transmisores, se plantean usos comprensivos/situacionales en determinados momentos, y hay una ausencia de recomendaciones sobre usos críticos de los diferentes recursos. 


\section{Referencias}

APPLE, M. W. (1986). Ideologia y Curriculo. Madrid, Akal.

CARR, W. y KemmisI, S. (1988). Teoria crítica de la enseñanza, Barcelona, Martínez Roca.

CONTRERAS, J. (1987). «De estudiante a profesor. Socialización y aprendizaje en las prácticas de enseñanza». Revista de Educación, 282, pp. 203-231.

DISEÑO CURRICULAR BASE. 1989. Educación Primaria, MEC.

DISEÑO CURRICULAR BASE. 1989. Educación Secundaria I. MEC.

GIMENO SACRISTÁN, J. (1983). «El profesor como investigador en el aula: un paradigma en la formación de profesores». Educación y Sociedad, 2, 51.73.

- (1988). El curriculum: una reflexión sobre la práctica. Madrid, Morata.

ELLIOT, J. (1986) . «Autoevaluación, desarrollo profesional y responsabilidad» en Galton, M. y Moon, B. Cambiar la escuela, cambiar el curriculum. Barcelona, Martínez Roca, 237-259.

HuLL, CH. (1986). «Cómo lograr la triangulación cuando sólo hay dos en el cuadrilátero». En Elliot, J. y otros Investigación, Acción en la Escuela, Valencia, Generalitat de Valencia.

KeMmis, S. (1988). El curriculum: más allá de la teoría de la reproducción, Madrid, Morata.

Perez Gomez, A. I. (1988). «El pensamiento práctico del profesor: implicaciones en la formación del profesorado». En Villa, A. (Coord.), Perspectivas y problemas de la función docente, Madrid, Narcea, 128-148.

Perez Gómez y Gimeno, J. (1988). «Pensamiento y acción en el profesor de los estudios sobre la planificación al pensamiento práctico». Infancia y Aprendizaje, 41, 37-63.

Peters, J. (1987). «La reflexión. Un concepto clave en la formación del profesor». Revista de Educación, 282, 191-201.

P.N.T.I.C. (1988). Proyectos Atenea y Mercurio. Secretaría de Estado de Educación. MEC.

P.N.T.I.C. (1989). Informe de progreso. Fase exploratoria (Proyecto Atenea). Secretaría de Estado de Educacón, MEC.

POPKEWITZ, T. S. (1987). «La producción del conocimiento escolar y los lenguajes curriculares. Cuestiones institucionales en el seguimiento de las matemáticas escolares». Revista de Educación, 282. 61-86.

PROYECTO ATENEA. Una propuesta para la introducción racional de las Nuevas Tecnologías de la Información en la Enseñanza Básica y Media (Versión resumida). Secretaría General Técnica. MEC, (1985).

Shulman, L. (1987). «Knowledge and Teaching: Foundations of the New Reform», Harvard Educational Review, 57, 1; 1-22.

TORRES, J. (1987-88). «Diseño del currículum». Nuestra Escuela, Diciembre-Enero, 7-12.

- (1989). «Libros de texto y control del currículum». Cuadernos de Pedagogía, 168, 50-55.

El uso de los medios desde las teorías del currículum. A. Bautista. CL\&E, 1989, 3-4, pp. 39-51.

\section{Resumen}

En este artículo se distinguen tres tipos de usos de los medios en la enseñanza. Los usos transmisores/reproductores están orientados por modelos del currículum cuyo diseño y desarrollo se realiza en torno a la definición de objetivos operativos y la selección y organización de contenidos, medios y materiales es realizada por las administraciones de educación de un país.

El segundo tipo de uso de medios, comprensión/situacional está orientado por modelos curriculares situacionales e interpretativos. Este uso supone un análisis y comprensión previa de los significados construidos por grupos concretos de profesores y alumnos. Los medios son usados como elementos que ayudan a representar y ejecutar planes que resuelven situaciones problemáticas y desarrollen proyectos de trabajo.

Finalmente, los usos críticos/transformadores de los medios están orientados por las teorias críticas del currículum. En este tercer tipo de uso, los medios se utilizan como elementos para analizar, reflexionar, criticar y transformar las prácticas de enseñanza y de mensajes que llegan al centro escolar y que son considerados nocivos por grupos de profesores a dicho centro.

\section{Datos sobre el autor:}

Antonio Bautista es profesor del Departamento de Didáctica en la Facultad de Filosofia y Ciencias de la Educación de la Universidad Complutense y lleva trabajando varios años en el uso pedagógico de los medios audiovisuales y del ordenador. 


\section{Dirección:}

Universidad Complutense de Madrid. Facultad de Filosofía y Ciencias de la Educación. Departamento de Didáctica y Organización escolar. Ciudad Universitaria, 28071 Madrid.

(C) de todos los artículos. Deberá solicitarse por escrito autorización de CL\&E y de los autores para el uso en forma de facsímil, fotocopia o cualquier otro medio de reproducción impresa. CL\&E se reserva el derecho de interponer las acciones legales necesarias en aquellos casos en que se contravenga la ley de derechos de autor.

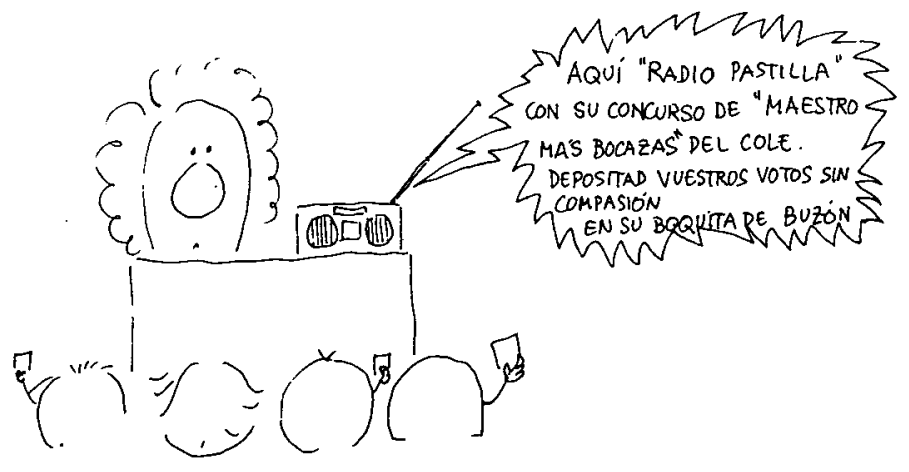

